\title{
Spin-Hybrids: A Single-Molecule Approach to Spintronics*
}

\author{
D. E. Bürgler, V. Heß, T. Esat, S. Fahrendorf, F. Matthes, and C. M. Schneider ${ }^{\dagger}$ \\ Peter Grünberg Institute PGI-6, Research Center Jülich, 52425 Jülich, Germany and
} JARA-FIT

\author{
C. Besson $\stackrel{\ddagger}{\ddagger}$ K. Yu. Monakhov, and P. Kögerler \\ Institute of Inorganic Chemistry, RWTH Aachen University, Landoltweg 1, 52074 Aachen, Germany \\ Peter Grünberg Institute PGI-6, Research Center Jülich, 52425 Jülich, Germany and
} JARA-FIT

A. Ghisolfi and P. Braunstein

Laboratoire de Chimie de Coordination, Institut de Chimie (UMR 7177 CNRS), Université de Strasbourg, 4 rue Blaise Pascal, 67081 Strasbourg, France

N. Atodiresei, V. Caciuc, and S. Blügel

Peter Grünberg Institute PGI-1, Research Center Jülich, 52425 Jülich, Germany and JARA-FIT

(Received 10 December 2015; Accepted 19 January 2016; Published 6 February 2016)

\begin{abstract}
Molecular spintronics aims at exploiting and controlling spin-dependent transport processes at the molecular level. Achieving this aim requires not only appropriate molecules, molecular structures and preparation procedures. Equally important is the understanding and engineering of the electronic and spin-dependent interactions between different molecular species, molecule and substrate, as well as molecule and electrodes. These interactions may not only determine the spin-dependent functionality of the molecular structures, but also their integrity on the substrate. Likewise, there may be also a modification of the surface properties below and in the vicinity of a molecule. We have investigated several molecules on different metallic surfaces, among them magnetic Nd doubledecker phthalocyanines, a cubane-type $\left\{\mathrm{Ni}_{4}\right\}$ complex with single-molecule magnet properties, and a nonmagnetic triazine-based molecule. For $\mathrm{NdPc}_{2}$ molecules adsorbed on a $\mathrm{Cu}(100)$ surface, our scanning tunneling microscopy and spectroscopy studies show specific electronic states of the molecule-substrate complex. We find that the electric field between STM tip and sample must be taken into account to properly describe the electronic states associated with the upper Pc ligand. [DOI: 10.1380/ejssnt.2016.17]
\end{abstract}

Keywords: Scanning Tunneling Microscopy; Density functional calculations; Chemisorption; Magnetic interfaces; Magnetic, structural, and other properties of nanostructures

\section{INTRODUCTION}

A continuously increasing energy demand in information technology asks for alternative state variables beyond the electron charge to encode digital information. Since the discovery of large spin-dependent electrical transport processes in the 1980's [1, 2], which laid the foundation to the field of "spintronics", the electron spin is considered as a potential pathway to energy-efficient storage, transfer and processing of data. Today, a reliable source of spin-polarized carriers is most often a magnetic metal or semiconductor. These materials which have a long-range ferro-, ferri-, or antiferromagnetic order intrinsically provide a "nonvolatility", as the digital information can be stored, e.g. in the local magnetization or spin direction of a small magnetic element.

A considerable challenge in the preparation of magnetic nanostructures, however, is the precise control of their magnetic properties once the dimensions reach the

\footnotetext{
* This paper was presented at the 10th International Symposium on Atomic Level Characterizations for New Materials and Devices '15, Shimane Prefectural Convention Center (Kunibiki Messe), Matsue, Japan, October 25-30, 2015.

$\dagger$ Corresponding author: c.m.schneider@fz-juelich.de

$\ddagger$ Present address: Department of Chemistry, The George Washington University, Washington, D.C., USA
}

$10 \mathrm{~nm}$ regime. Small changes in the size or shape of the elements may lead to significant changes in magnetic anisotropies, switching fields or switching character. This problem can in principle be circumvented by employing single-molecule magnets, if these molecules carry a large magnetic moment and have a sufficiently high magnetic anisotropy to preserve the spin orientation in space. The main advantage of a chemical synthesis is the production of a large number of molecules with identical magnetic properties. A magnetic molecule may serve as an entity to store digital information or as spin-polarized electrode in a two- or three-terminal molecular electronic device [3]. Moreover, the spin state of a certain class of molecules spin crossover compounds - may be controlled by external stimuli, such as light [4]. This explains the recent interest in the areas of "molecular magnetism" and "molecular spintronics".

At present, however, approaches in molecular spintronics are limited to cryogenic temperatures, as thermal fluctuations can easily change the magnetization direction in a single molecule. A solution is the construction of molecular hybrid systems - spin-hybrids -, in which the magnetization of the single molecular magnet is stabilized by exchange coupling to another magnetic system $[5,6]$. In order to control and taylor the physical properties of these spin-hybrids, we have to first understand of the electronic interactions and charge transfer mechanisms between molecule and substrate. These determine 

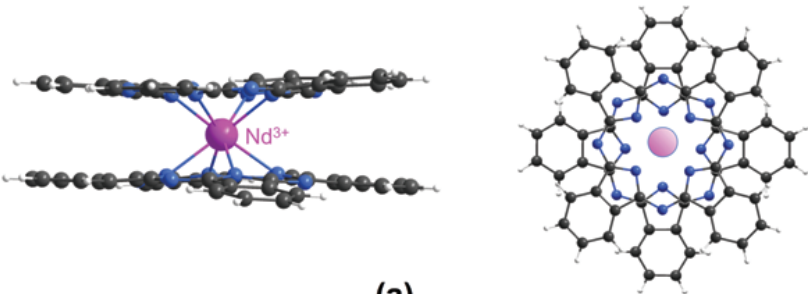

(a)
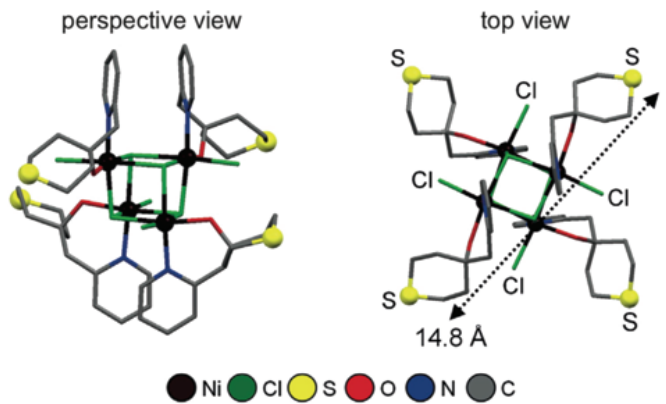

(b)
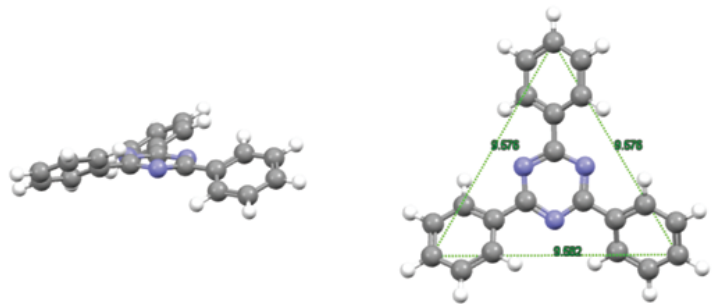

(c)

FIG. 1. Graphical representation of the molecules studied. (a) $\mathrm{Nd}$ double-decker phthalocyanine molecule $\mathrm{NdPc}_{2}$. (b) Cubane-type $\left\{\mathrm{Ni}_{4}\right\}$ complex. Hydrogen atoms have been omitted for clarity. (c) 2,4,6-triphenyl-1,3,5-triazine (TPT).

not only the strength and character of the exchange coupling, but may even affect the chemical stability of the molecule in contact with the substrate. The opportunity to specifically exploit and to engineer these interactions makes hybrid spin systems a new class of magnetic materials, which promises unique magnetic properties and novel spin-dependent functionalities [7-9].

In the following, we will discuss experiments carried out with three different classes of molecules: (i) a magnetic molecule containing a $4 f$ metal ion center, (ii) a singlemolecule magnet (SMM) containing $3 d$ metal ion centers, and (iii) a metal-ion free molecule.

\section{DEPOSITION OF MAGNETIC MOLECULES}

In our studies we employ several deposition techniques, which depend on the stability of the molecules under investigation. A strict surface-science approach, i.e. a deposition of the molecules onto the substrate by thermal evaporation under ultrahigh vacuum conditions is desirable, but not always feasible. Many interesting classes of molecules do not possess a sufficient volatility and/or thermal stability and fragment during thermal evapora- tion. In the following we focus on two types of magnetic molecules, which have been specifically synthesized in-house for our experiments.

The first one comprises a neodymium double-decker phthalocyanine $\left(\mathrm{NdPc}_{2}\right)$. The structure consists of two phthalocyanine rings which are connected via a central Nd atom resulting in an hourglass shaped molecule (Fig. 1(a)). This molecule was deposited onto $\mathrm{Cu}(100)$, $\mathrm{Au}(111)$ and $\mathrm{Fe}(110)$ by means of molecular beam epitaxy at $800 \mathrm{~K}$ source temperature $[10,11]$.

The second molecule is a cubane-type tetranuclear $\mathrm{Ni}(\mathrm{II})$ complex with neutral, thioether-functionalized ligands. We want to explore the selective binding of novel sulfur-containing end groups to a Au surface (Fig. 1(b)). The molecule contains four pyridyl-alcohol-type ligands with structurally exposed, peripheral thio-cyclohexane functionalities. The four Ni centers exhibit a ferromagnetic coupling. $\left\{\mathrm{Ni}_{4}\right\}$ thin films in the monolayer (ML) regime were deposited ex-situ by immersing the previously cleaned $\mathrm{Au}(111)$ crystal surface into an oxygen-free $0.5 \mathrm{mM}\left\{\mathrm{Ni}_{4}\right\}$-dichloromethane ( $\left.\mathrm{DCM}, \mathrm{CH}_{2} \mathrm{Cl}_{2}\right)$ solution, followed by an immersion in pure DCM to remove unbound species and contaminations. The deposition was conducted under argon atmosphere and at room temperature [12].

The third molecule is an aromatic compound without metal centers. It is a commercially available triphenyltriazine complex (2,4,6-triphenyl-1,3,5-triazine) consisting of a central triazine ring and three phenyl groups (Fig. 1(c)). The molecule has a three-fold symmetry and will be henceforth denoted TPT for reasons of simplicity. TPT in the gas phase is non-magnetic. The deposition of the TPT molecules was carried out with molecular beam epitaxy under UHV conditions onto $\mathrm{Fe}(011)$ and $\mathrm{Co}(111)$ surfaces held at $170 \mathrm{~K}$ for $\mathrm{Fe}$ and below $70 \mathrm{~K}$ for Co, respectively. The motivation to study non-magnetic, aromatic molecules on ferromagnetic surfaces like $\mathrm{Fe}$ or $\mathrm{Co}$ is driven by the desire to understand the spin-polarized hybridization between the $3 d$-states of the metal with the $\pi$-orbitals of the molecule. On the one hand, this results in a spin-unbalanced electronic structure in the chemisorbed molecule giving rise to an induced molecular magnetic moment or spin-filter functionality $[9,13]$. On the other hand, the hybridization also modifies the electronic and magnetic properties of the metal atoms that are binding directly to the molecule. The strength of the exchange interaction among these atoms is locally enhanced compared to the clean surface. The consequence is a magnetic hardening effect that leads to the formation of a novel magnetic unit called hybrid molecular magnet consisting of the chemisorbed molecule and its immediate metal neighbors [9]. First experiments on hybrid molecular magnets [8] reveal interface magnetoresistance response up to $250 \mathrm{~K}$, possibly demonstrating a route to molecular spintronics at room temperature.

\section{STABILITY OF CHEMISORBED MOLECULES}

The first issue in spin hybrids that one has to deal with is the stability of a molecule during the deposition and adsorption onto a surface or an electrode. The stability 

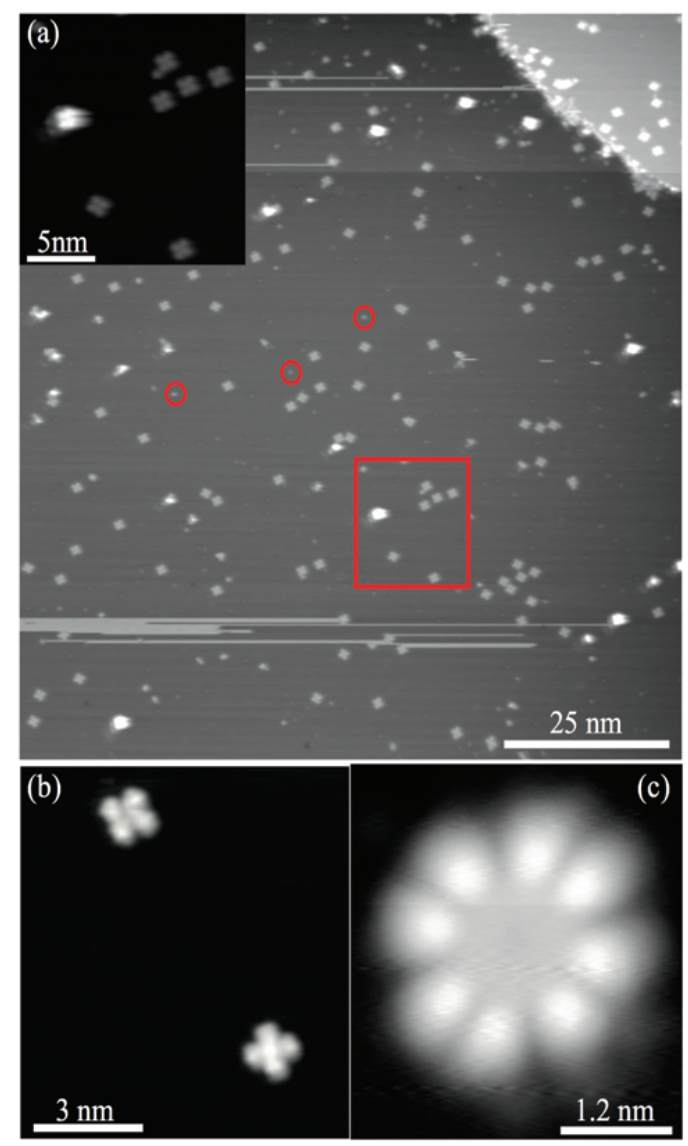

FIG. 2. STM images taken after $\mathrm{NdPc}_{2}$ deposition on $\mathrm{Cu}(100)$ : (a) Overview showing single- and double-decker Pc, and some defects (circles). (b) Single- and (c) double-decker Pc molecule.

is sensitive to the details of the electronic interactions in the molecule-surface system and the molecule itself.

The main methods employed in this work were scanning tunneling microscopy (STM) and scanning tunneling spectroscopy (STS). We used two types of lowtemperature STM systems, both of which where also equipped with sample preparation and surface characterization facilities. The first instrument comprised an Omicron LT-STM capable of reaching a base temperature of around $5 \mathrm{~K}$. The second instrument was a SPECS Joule-Thomson STM which can reach a minimum sample temperature of $1.2 \mathrm{~K}$ with the Joule-Thomson stage. In addition, in this STM we can apply a magnetic field of up to $\pm 3 \mathrm{~T}$ normal to the sample surface. Both instruments have already been shown to also work in a spin-polarized mode by using ferromagnetic or antiferromagnetic tips.

The late-lanthanide phthalocyanines, for example, $\mathrm{TbPc}_{2}$ have been investigated quite extensively in the past and have been found to be stable on many surfaces [1418]. This is not necessarily true for the early-lanthanide phthalocyanines. The data from scanning tunneling microscopy reproduced in Fig. 2 show a submonolayer coverage of $\mathrm{NdPc}_{2}$ molecules on $\mathrm{Cu}(100)$. The adsorbed molecules give rise to basically two kinds of structures, which differ by the contrast and the shape of the pattern. We find structures with four symmetric lobes, and structures with eight lobes. We identify the former with single

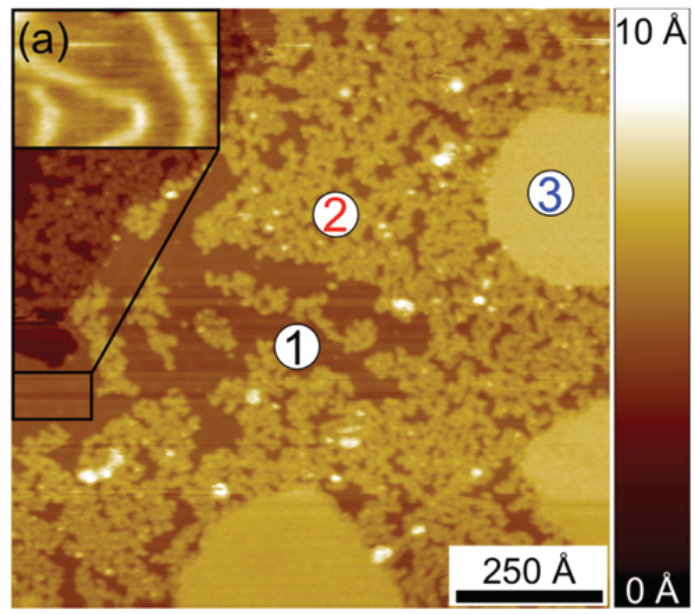

FIG. 3. Surface morphology after $\left\{\mathrm{Ni}_{4}\right\}$ deposition and postannealing at $590 \mathrm{~K}$. (a) STM image at $5 \mathrm{~K}$ showing uncovered (1), disordered (2), and ordered (3) areas. The numbers are placed exemplarily on corresponding areas. Inset: A distorted herringbone reconstruction appears in uncovered areas.

$\mathrm{Pc}$ rings resulting from a decomposition of the molecules, whereas the latter structure is a signature of intact $\mathrm{NdPc}_{2}$ entities. In fact, only about $20 \%$ of the deposited doubledecker Pc's remain intact upon deposition onto $\mathrm{Cu}(100)$.

The comparison between deposition onto $\mathrm{Cu}(100)$, $\mathrm{Au}(111)$ and $\mathrm{Fe}(110)$ yields a surprising result [11]. The $\mathrm{NdPc}_{2}$ molecule is more stable on nominally more reactive surfaces, such as $\mathrm{Fe}(110)$, where about $50 \%$ of the molecules remain intact. By contrast, on the inert $\mathrm{Au}(111)$ surface, all molecules decompose upon chemisorption. On the one hand, this is a clear indication that the molecule-surface interaction of chemisorbed species is very important and affects the intramolecular bonding, leading to a decomposition of the double-decker in two Pc rings. On the other hand, the result is counterintuitive. Estimating the adsorption energies of the molecule from comparison to benzene rings, one should expect the highest adsorption energy for the Fe(110) surface. The experiments, however, show the least tendency for decomposition for the strongest chemisorption. We attribute these findings to a substrate-dependent partial charge transfer from the substrate to the Pc ligands of the molecule. This charge transfer strengthens the intramolecular bonding mediated predominantly by electrostatic interaction. This interpretation is supported by studies of the binding energy separation of the bonding and antibonding $4 f$-states (ligand-field splitting) by means of scanning tunneling spectroscopy (STS). We find an increase of the splitting on Fe(110) which marks an increased intramolecular bonding.

The cubane-type $\left\{\mathrm{Ni}_{4}\right\}$ complex in the crystallized state is thermally stable up to $525 \mathrm{~K}$. In literature the formation of highly ordered self-assembled monolayers (SAMs) with thioether-functionalized molecules has been reported $[19,20]$. Our STM studies do not show, however, a periodic surface morphology related to ordered SAMs, but rather an almost featureless surface. In fact, the results provide strong evidence for partial decomposition of the coordination complex upon deposition on the 

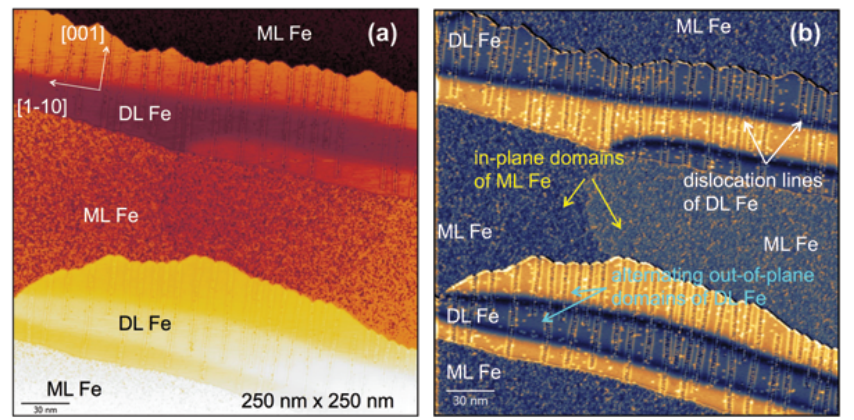

FIG. 4. Spin-polarized STM data from Fe/W(110) taken at 4 $\mathrm{K}$ with a Cr-coated STM tip. (a) Topography image showing monolayer (ML) and double-layer (DL) stripes. (b) $d I / d V$ map taken at a tunneling voltage of $-100 \mathrm{mV}$ showing inplane and out-of-plane magnetization directions for ML and DL stripes, respectively.

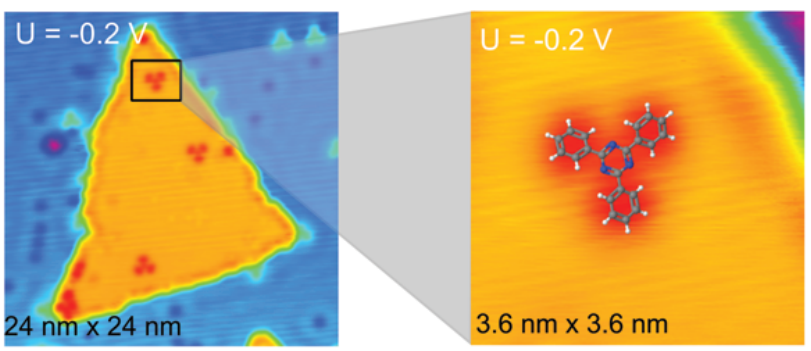

FIG. 5. STM data from TPT molecules deposited on Co(111) islands. The enlarged area has been overlaid with a sketch of the TPT molecule to illustrate the symmetry of the contrast pattern.

$\mathrm{Au}(111)$ surface [12]. Spectroscopic investigations confirm, however, that the magnetic $\mathrm{Ni}_{4} \mathrm{Cl}_{4 n}(n=1$ or 2$)$ core remains intact upon chemisorption. The fragments resulting from the decomposition of the $\left\{\mathrm{Ni}_{4}\right\}$ complex are lying in a disordered fashion on the surface, giving rise to the diffuse STM morphology.

The magnetic $\mathrm{Ni}_{4} \mathrm{Cl}_{4 n}$ core on $\mathrm{Au}(111)$ remains stable up to ca. $480 \mathrm{~K}$. This suggests that such a type of coordination complexes can be tuned to retain their SMM properties exhibited in the bulk [21] as long as the local molecular electrostatic environment does not significantly influence the molecular magnetic anisotropy. Only postannealing of the films up to $590 \mathrm{~K}$ induces further decomposition and fragmentation of the $\mathrm{Ni}_{4} \mathrm{Cl}_{4 n}$ core. The respective STM micrographs reveal uncovered $\mathrm{Au}(111)$ areas with a distorted herringbone reconstruction, as well as disordered regions and areas with some structural order (Fig. 3). The detailed insight into the chemisorptioninduced decomposition pathway provides guidelines for the future deposition of thioether-functionalized $\mathrm{Ni}(\mathrm{II})$ complexes on metallic surfaces.

\section{FIRST RESULTS ON TPT MOLECULES}

The TPT molecules are deposited onto ferromagnetic substrates which consist of ultrathin layers of Fe/W(110)

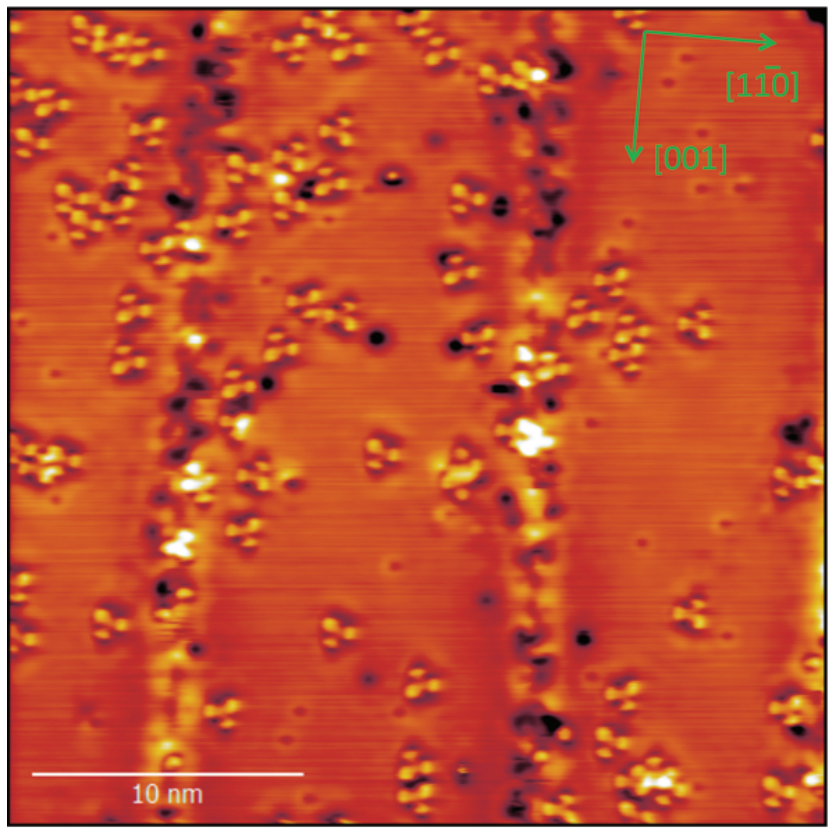

FIG. 6. STM data from TPT molecules deposited on Fe(110) stripes with 2 monolayer thickness. The vertical lines are caused by the dislocation lines.

and $\mathrm{Co} / \mathrm{Cu}(111)$. In the case of the Fe films, approximately 2 monolayers are grown at room temperature then postannealed at $500 \mathrm{~K}$. This leads to the formation of a particular morphology with alternating stripes of $1 \mathrm{ML}$ and $2 \mathrm{ML}$ height (Fig. 4(a)). In addition to the stripe structure, there is a characteristic pattern of dislocation lines which forms on the double layer stripes. By using a Cr coated W tip, the same surface region has been imaged in a spin-resolved mode revealing the local magnetization direction in the stripes. We find in-plane magnetic domains in the 1 ML stripes, whereas the 2 ML stripes exhibit an out-of-plane magnetization direction (Fig. 4(b)). This is in agreement with earlier observations $[22,23]$.

The second ferromagnetic substrate consists of Co grown on $\mathrm{Cu}(111)$ at room temperature. This system is known to exhibit an island growth due to the formation of stacking faults. At submonolayer coverages, the Co atoms assemble in triangular islands which are known to be magnetized out-of-plane.

The deposition of a submonolayer coverage of TPT on $\mathrm{Co}(111)$ has been performed at low temperature. The STM micrographs reveal clear localized contrast patterns with a three-fold symmetry both on the bare $\mathrm{Cu}(111)$ surface and the $\mathrm{Co}(111)$ islands (Fig. 5). This contrast pattern resembles closely the geometry of the TPT molecule. This can be seen by an enlarged image of the pattern. A graphical representation of the TPT molecule has been overlaid to the contrast for illustration purposes. As we do not find signatures of fragments, we have to assume that most of the molecules stay intact after the deposition. We note that there are two adsorption orientations of the molecule, which are clearly visible in the experimental data. Considering the symmetries of the fcc (111) surface and the TPT molecule, we should expect 6 different adsorption geometries which differ by subsequent 


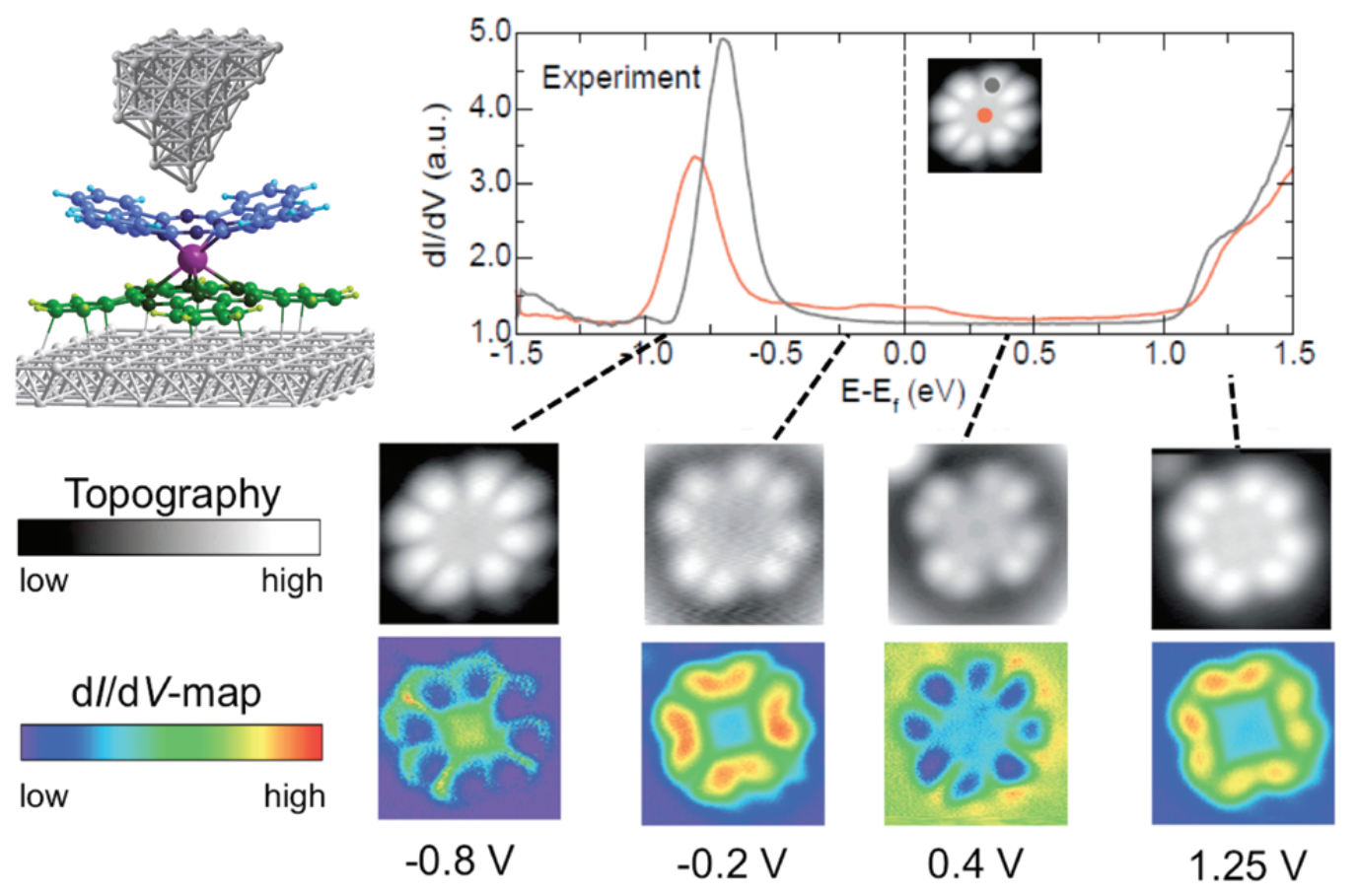

FIG. 7. STS data from $\mathrm{NdPc}_{2}$ molecules on $\mathrm{Cu}(100)$. Top: $d I / d V$ spectra taken at different locations on the molecule. Bottom: topography and $d I / d V$ maps taken at selected binding energies reflecting the characteristic 8-lobed pattern of an intact $\mathrm{NdP}_{2}$ molecule.

rotations of $60^{\circ}$, but are three-fold degenerate.

On the Fe(110) stripes the TPT molecule has been deposited at $170 \mathrm{~K}$, while the subsequent STM studies were again carried out at low temperature. The data shown in (Fig. 6) have been obtained from a 2 ML stripe. We see the typical three-fold symmetric contrast patterns due to the TPT molecules. Although there is a certain tendency for the molecules to aggregate at the dislocation lines, there are also numerous molecules in between the lines. It also appears that the molecules stay mainly intact during the deposition. A closer inspection of the contrast patterns reveals the presence of at least two different adsorption orientations.

The above results show that for both ferromagnetic surfaces the TPT molecules mainly stay intact upon deposition, adsorb in a well-ordered manner, and strongly interact (hybridize) with the substrate. This forms the basis for future spin-resolved STM studies to elucidate the electronic and magnetic properties of the TPT/Fe and TPT/Co hybrid molecular magnets.

\section{ELECTRONIC STRUCTURE IN $\mathrm{NdPc}_{2} / \mathrm{Cu}(\mathbf{1 0 0})$}

In order to elucidate the electronic structure of the $\mathrm{NdP}_{2}$ molecules deposited on $\mathrm{Cu}(100)$ we used scanning tunneling spectroscopy with atomic resolution. We record energy-dependent topography and differential conductivity $(d I / d V)$ maps as well as $d I / d V(V)$ curves at different sites on the $\mathrm{NdPc}_{2}$ molecule (see Fig. 7). In an effort to interpret the STS results, we performed extensive DFT calculations which also took into account the van der Waals interactions [10]. The spatially and energetically resolved orbital maps compare well with the predictions from DFT. This allows us to identify them with specific electronic states of the molecule-substrate complex. As a result of the combined experiment/theory approach we find that the bottom Pc ring strongly hybridizes with the substrate resulting in a bonding to the substrate. The upper Pc ring keeps its molecular-type electronic states, which are reflected in the HOMO and LUMO contributions to the $d I / d V$ spectra. In addition, we observe direct spectral signatures of the spin-split Nd $4 f$-states.

The strong difference between the electronic behavior of the bottom and top $\mathrm{Pc}$ ring gives rise to a particular phenomenon. The upper Pc ligand is barely coupled to the substrate and the DFT calculations predict that the molecular-like electronic states are highly susceptible to an electric field. In fact, we find that the electric field between the STM tip and sample must be taken into account to properly describe the energy position of the electronic states associated with the upper Pc ligand. With increasing field strength these states shift for both field polarities away from the Fermi level until they hybridize with the Nd $4 f$-orbitals, where they are pinned and can then be measured in STS [10].

Furthermore, we demonstrate for the first time that the spin-polarized $\mathrm{Nd} 4 f$-states are involved in the charge transport through $\mathrm{NdPc}_{2}$ on $\mathrm{Cu}(100)$ [10]. In contrast to the $\mathrm{Tb}^{3+}$ ion in the previously studied late-Lanthanide analogue $\mathrm{TbPc}_{2}[15,18]$, the $\mathrm{Nd}^{3+}$ ion has a larger ionic radius with more delocalized $4 f$-electrons. As a consequence, the $4 f$-electrons undergo a stronger hybridization with the $\mathrm{Pc}$ ligands. Thereby, the $4 f$-orbitals become accessible to transport experiments, while largely maintaining the relative energetic positions of spin-up/down states and hence the magnetic moment [10]. This 
result opens up prospects for electrical manipulation and detection of the molecular spin state, providing the basis for all-electrically controlled device concepts in molecular spintronics.

\section{CONCLUSION}

The recent developments in the chemical synthesis procedures provide many opportunities to create novel singlemolecule magnet systems. Our studies show that a successful incorporation of magnetic molecules into a spintronic structure requires a careful investigation of the interactions between the molecule and environment. It is important to realize that the surface and the adsorbed molecule form a true spin hybrid system. On the one hand, these interactions may considerably change the properties of a magnetic molecule as compared to behavior in solution or in the crystallized state. On the other hand, the interaction with the molecule may also change the magnetic behavior of the surface in the vicinity of the molecule. Controlling and tailoring these interactions will open up unique opportunities to create hybrid systems with novel spin-dependent properties.

\section{ACKNOWLEDGMENTS}

C.B. thanks the Alexander von Humboldt Foundation for a fellowship. K.Y.M. thanks the Excellence Initiative of the German federal and state governments for an RWTH Start-Up grant. A.G. and P.B. are grateful to the CNRS, the Ministère de la Recherche (Paris), the DFH/UFA (International Research Training Group GRK532, Ph.D. grant to A.G.), and the International Centre for Frontier Research in Chemistry, Strasbourg (icFRC, http://www.icfrc.fr). The computations were performed under the auspices of the VSR at the computer JUROPA and the Gauss Centre for Supercomputing at the high-performance computer JUGENE operated by the Jülich Supercomputer Centre at the Forschungszentrum Jülich.
[1] M. N. Baibich, J. M. Broto, A. Fert, F. N. V. Dau, F. Petroff, P. Etienne, G. Creuzet, A. Friederich, and J. Chazelas, Phys. Rev. Lett. 61, 2472 (1988).

[2] G. Binasch, P. Grünberg, F. Saurenbach, and W. Zinn, Phys. Rev. B 39, 4828 (1989).

[3] S. Schmaus, A. Bagrets, Y. Nahas, T. K. Yamada, A. Bork, M. Bowen, E. Beaurepaire, F. Evers, and W. Wulfhekel, Nat. Nanotechnol. 6, 185 (2011).

[4] A. Bousseksou, G. Molnár, L. Salmon, and W. Nicolazzi, Chem. Soc. Rev. 40, 3313 (2011).

[5] H. Wende, M. Bernien, J. Luo, C. Sorg, N. Ponpandian, J. Kurde, J. Miguel, M. Piantek, X. Xu, P. Eckhold, W. Kuch, K. Baberschke, P. M. Panchmatia, B. Sanyal, P. M. Oppeneer, and O. Eriksson, Nat. Mater. 6, 516 (2007).

[6] M. Bernien, J. Miguel, C. Weis, M. E. Ali, J. Kurde, B. Krumme, P. M. Panchmatia, B. Sanyal, M. Piantek, P. Srivastava, K. Baberschke, P. M. Oppeneer, O. Eriksson, W. Kuch, and H. Wende, Phys. Rev. Lett. 102, 047202 (2009).

[7] T. Miyamachi, M. Gruber, V. Davesne, M. Bowen, S. Boukari, L. Ø. Joly, F. Scheurer, G. Rogez, T. K. Yamada, P. Ohresser, E. Beaurepaire, and W. Wulfhekel, Nat. Commun. 3, 938 (2012).

[8] K. V. Raman, A. M. Kamerbeek, A. Mukherjee, N. Atodiresei, T. K. Sen, P. Lazic, V. Caciuc, R. Michel, D. Stalke, S. K. Mandal, S. Blügel, M. Münzenberg, and J. S. Moodera, Nature 493, 509 (2013).

[9] M. Callsen, V. Caciuc, N. Kiselev, N. Atodiresei, and S. Blügel, Phys. Rev. Lett. 111, 5 (2013).

[10] S. Fahrendorf, N. Atodiresei, C. Besson, V. Caciuc, F. Matthes, S. Blügel, P. Kögerler, D. E. Bürgler, and C. M. Schneider, Nat. Commun. 4, 2425 (2013).

[11] S. Fahrendorf, F. Matthes, D. E. Bürgler, C. M. Schneider, N. Atodiresei, V. Caciuc, S. Blügel, C. Besson, and
P. Kögerler, SPIN 4, 1440007 (2014)

[12] V. Heß, F. Matthes, D. E. Bürgler, K. Y. Monakhov, C. Besson, P. Kögerler, A. Ghisolfi, P. Braunstein, and C. M. Schneider, Surf. Sci. 641, 210 (2015).

[13] M. Galbiati, S. Tatay, C. Barraud, A. V. Dediu, F. Petroff, R. Mattana, and P. Seneor. MRS Bulletin 39, 602 (2014).

[14] L. Vitali, S. Fabris, A. M. Conte, S. Brink, M. Ruben, S. Baroni, and K. Kern. Nano Lett. 8, 3364 (2008).

[15] T. Komeda, H. Isshiki, J. Liu, Y.-F. Zhang, N. Lorente, K. Katoh, B. K. Breedlove, and M. Yamashita, Nat. Commun. 2, 217 (2011).

[16] S. Stepanow, J. Honolka, P. Gambardella, L. Vitali, N. Abdurakhmanova, T.-C. Tseng, S. Rauschenbach, S. L. Tait, V. Sessi, S. Klyatskaya, M Ruben, and K. Kern, J. Am. Chem. Soc. 132, 11900 (2010).

[17] A. Lodi Rizzini, C. Krull, T. Balashov, J. J. Kavich, A. Mugarza, P. S. Miedema, P. K. Thakur, V. Sessi, S. Klyatskaya, M. Ruben, S. Stepanow, and P. Gambardella. Phys. Rev. Lett. 107, 177205 (2011).

[18] J. Schwöbel, Y. Fu, J. Brede, A. Dilullo, G. Hoffmann, S. Klyatskaya, M. Ruben, and R. Wiesendanger, Nat. Commun. 3, 953 (2012).

[19] J. Noh, T. Murase, K. Nakajima, H. Lee, and M. Hara, J. Phys. Chem. B 104, 7411 (2000).

[20] E. B. Troughton, C. D. Bain, G. M. Whitesides, R. G. Nuzzo, D. L. Allara, and M. D. Porter, Langmuir 4, 365 (1988).

[21] A. Ghisolfi, K. Y. Monakhov, R. Pattacini, P. Braunstein, X. López, C. de Graaf, M. Speldrich, J. van Leusen, H. Schilder, and P. Kögerler. Dalton Trans. 43, 7847 (2014).

[22] O. Pietzsch, A. Kubetzka, M. Bode, and R. Wiesendanger, Phys. Rev. Lett. 84, 5212 (2000).

[23] A. Kubetzka, M. Bode, O. Pietzsch, and R. Wiesendanger, Phys. Rev. Lett. 88, 057201 (2002). 